\title{
Prevalencia de actividad física y beneficios y barreras en trabajadores de Villavicencio, Colombia
}

\author{
Prevalence of physical activity and of benefits and \\ barriers in workers of Villavicencio, Colombia
}

\author{
Johao-Alexander Colmenares-Pedraza ${ }^{1}$; Rodolfo Herrera-Medina ${ }^{2}$
}

Forma de citar: Colmenares Pedraza JA, Herrera Medina R. Prevalencia de actividad física y beneficios y barreras en trabajadores de Villavicencio, Colombia. Rev Univ Ind Santander Salud. 2018; 50(1): 37-45. doi: http://dx.doi.org/10.18273/revsal.v50n1-2018004@亦

\section{Resumen}

Introducción: El acelerado ritmo de vida de los trabajadores los expone al incremento en los niveles de sedentarismo, influenciados por factores sociodemográficos, biológicos, económicos y ambientales que a su vez aumentan el riesgo de desarrollar enfermedades crónicas. Objetivo: Determinar la prevalencia de la práctica de actividad física global, beneficios y barreras psicológicas y de salud en trabajadores de una empresa de promoción de seguridad social y recreación de Villavicencio durante el primer trimestre del 2017. Metodología: Estudio observacional, analítico de corte transversal. Se empleó la versión corta del Cuestionario Internacional de Actividad Física IPAQ, el cuestionario Physical Activity Readiness PAR-Q y la escala de percepción de beneficios y barreras para la práctica de actividad física. Resultados: La prevalencia de actividad física (moderada y vigorosa) fue 33,7\% ( $\mathrm{IC}_{95 \%}$ : 28,1-39,6\%). Las principales barreras de salud consistieron en la presencia de problemas osteoarticulares, mareo y dolor precordial al realizar ejercicio. La percepción alta de beneficios y barreras psicológicas fue de $92,8 \%$ y $6,9 \%$, respectivamente. Los principales beneficios psicológicos percibidos estuvieron relacionados con la salud y la autopercepción de la imagen, mientras que las principales barreras fueron fatiga, cansancio y falta de tiempo. La oportunidad de ser inactivo físicamente en mujeres fue dos veces mayor respecto a los hombres. Conclusiones: Existe baja prevalencia de práctica de actividad física en la población estudiada, sobre todo en mujeres. Los beneficios psicológicos estuvieron orientados a la percepción del mejor estado de salud y de la imagen corporal y las principales barreras identificadas fueron agotamiento físico y falta de tiempo.

Palabras clave: Ejercicio; Trabajador; Adulto; Promoción de la salud; Estudio observacional; Prevalencia.

\begin{abstract}
Introduction: The fast pace of life of workers exposes them to the increase in sedentary levels, which are influenced by sociodemographic, biological, economic and environmental factors that represent a higher risk of developing chronic diseases. Objective: To determine the prevalence of the practice of global physical activity and of psychological and health benefits and barriers in workers of a company of promotion of social security and

1. Universidad de los Llanos Orientales. Villavicencio, Colombia.

2. Universidad Libre seccional Cali. Valle del Cauca, Colombia.

Correspondencia: Johao Alexander Colmenares Pedraza. Dirección: Calle 38 No. 17B-61 Santa Helena, Villavicencio (Colombia). Correo electrónico: jcolmenares@unillanos.edu.co. Teléfono: +7 3173774465.
\end{abstract}


recreation of Villavicencio during the first quarter of 2017. Methodology: Cross-sectional, observational study. The short version of the International Physical Activity Questionnaire, the Physical Activity Readiness Questionnaire and the scale of perception of benefits and barriers to physical activity practice were used. Results: The prevalence of physical activity (moderate and vigorous) was 33.7\% (CI 95\%: 28.1-39.6\%). The main health barriers were to present osteoarticular problems, dizziness and precordial pain when exercising. The high perception of benefits and psychological barriers were $92.8 \%$ and $6.9 \%$, respectively. The main psychological benefits perceived were those related to health and self-perception of the image, while the main barriers were fatigue and lack of time. The opportunity of being physically inactive in women was two times greater than in men. Conclusions: There is a low prevalence of physical activity in the studied population, especially in women. The psychological benefits were oriented to the perception of a better state of health and of the corporal image and the main barriers identified were physical exhaustion and lack of time.

Keywords: Exercise; Worker; Adult; Health promotion; Observational study; Prevalence.

\section{Introducción}

Actualmente Colombia hace parte de los países en donde coexisten problemas de salud derivados de enfermedades infecciosas, característicos de países en vías de desarrollo con enfermedades ocasionadas por estilos de vida no saludables propios de países desarrollados. Es así como a partir de la década de los años 90 se ha visto un incremento en la presencia de factores de riesgo provenientes del comportamiento de las personas tales como la inactividad física, la cual junto con otro tipo de conductas como la alimentación inadecuada y el consumo de bebidas alcohólicas y de tabaco ha generado un aumento en más del $10 \%$ en la incidencia de enfermedades crónicas no transmisibles (ECNT) para el año 2010 ${ }^{1}$. De acuerdo con la Organización Mundial de la Salud (OMS) el sedentarismo es responsable de aproximadamente 3,2 millones de muertes por año e incrementa en un 20-30\% el riesgo de morir por todas las causas en la población de adultos trabajadores ${ }^{2}$.

Con respecto a la proporción de sedentarismo en la población adulta trabajadora, en el país se han realizado varios estudios: el programa Muévete Bogotá reportó un nivel de $47 \%$ de inactividad física en la población trabajadora de la capital; el estudio de enfoque multinivel para el diagnóstico de la actividad física en tres regiones del país mostró una prevalencia de sedentarismo de 59,3\%; de acuerdo a la última Encuesta Nacional de la Situación Nutricional en Colombia (ENSIN 2010), la prevalencia reportada de inactividad física en individuos de 18 a 64 años fue de 53,5\% $\%^{3-5}$.

En este sentido, los trabajadores constituyen una población cuya jornada laboral los expone a una variedad de situaciones, dentro de las cuales se encuentra la disminución en la cantidad y la calidad de su tiempo libre. Ante la imposibilidad de emplear de manera activa estos periodos de descanso, con el paso del tiempo se va generando una respuesta corporal negativa que impacta la salud y altera la vida familiar y personal del trabajador ${ }^{6}$.

Con relación a las consecuencias generadas, Martínez, et al. $^{7}$ en su estudio concluyeron que la inactividad física aumenta la frecuencia y duración de las tasas de incapacidad por enfermedad respiratoria y osteomuscular, lo cual conlleva a implicaciones desfavorables para el trabajador y la empresa. Hammer, et $\mathrm{al}^{8}$ encontraron una correlación positiva entre la práctica de actividad física y la salud mental. Ramos Piñero ${ }^{9}$ en su revisión publicada en el 2007 concluye como la inactividad física constituye un factor de riesgo para la mayoría de las enfermedades crónicas en la población económicamente activa, por lo que dicha condición representa una amenaza para la salud de los trabajadores y la productividad de las empresas. En el estudio de Hoffmeister, et al. ${ }^{10}$ se concluyó que una conducta sedentaria incrementó el riesgo de presentar un accidente laboral en la muestra de empleados estudiada.

En el contexto normativo nacional el Decreto 1295 de 1994 menciona la "responsabilidad indelegable del empleador en la necesidad de invertir en actividades que promuevan la salud en el trabajo"; La Ley 181 de 1995 menciona que las empresas con más de 50 trabajadores deben programar eventos deportivos directamente o mediante convenio con las Cajas de compensación y/o entidades especializadas. La ley define a una Caja de compensación familiar, como una corporación que desarrolla actividades de seguridad social y programas de servicios de prevención y promoción de la salud a empleados y afiliados ${ }^{11-14}$.

Partiendo de lo anteriormente descrito y tomando en cuenta la escasez de estudios de carácter descriptivo en Colombia se desarrolló una investigación que buscó 
estimar la prevalencia de la práctica de actividad física y de los beneficios y barreras psicológicas y de salud física percibidas en la población de trabajadores de una Caja de compensación en la ciudad de Villavicencio durante el primer trimestre del año 2017, con el fin de suministrar una base sustentada científicamente que permita el desarrollo de estudios de búsqueda de factores de asociación que fundamenten el diseño de los programas destinados a la promoción de la actividad física diseñados por la Caja de compensación.

\section{Metodología}

Se desarrolló un estudio observacional, analítico, de corte transversal.

La población estudio estuvo conformada por los trabajadores de la Caja de compensación familiar residentes en la ciudad de Villavicencio durante el primer trimestre del año 2017. Los criterios de inclusión fueron ser mayor de edad, trabajador de la Caja de compensación con mínimo un mes de antigüedad, residencia permanente durante los últimos tres meses en la ciudad de Villavicencio. Los criterios de exclusión fueron ser mujer gestante y aquellos con alguna limitación psicológica, mental y/o comunicativa para responder la encuesta.

El tamaño de muestra se calculó a partir de una población de 730 adultos $^{15}$, una proporción esperada de práctica de actividad física de $53,5 \%{ }^{5}$, un nivel de confianza de $95 \%$, un error máximo permitido de 5\% y una proporción adicional por no respuesta del $10 \%$. Para la selección de los participantes se empleó un muestreo aleatorio simple.

La recolección de la información se realizó a partir de una encuesta cerrada mediante una entrevista estructurada de una duración aproximada de 20 minutos y realizada en los sitios de trabajo de los participantes; conformada por una encuesta sociodemográfica tomada de la ENSIN 20105, la versión corta del Cuestionario Internacional de Actividad física IPAQ ${ }^{16}$, el cuestionario Physical Activity Readiness PAR-Q ${ }^{17}$ y la escala de percepción de beneficios y barreras para la práctica de actividad física ${ }^{18}$, los cuales han demostrado a través de estudios una adecuada validez y reproducibilidad ${ }^{17-24}$.

Para el cálculo del nivel de actividad física practicado (variable de interés) de acuerdo al IPAQ un total inferior a los 600 METs correspondieron a un nivel leve de actividad física, un total de 600 a 1499 METs a un nivel moderado y un total de 1500 METs y más a un nivel vigoroso. Las variables tenidas en cuenta en la encuesta sociodemográfica fueron edad, sexo, nivel de escolaridad, lugar de residencia, estrato de la vivienda, estado civil, composición de su hogar, número de personas que integran el hogar y tipo de hogar en el que vive. El PAR-Q, compuesto por siete preguntas con opciones de respuesta si/no, indagó sobre la presencia de algunas situaciones relacionadas con el estado de salud que podrían incidir en la práctica de ejercicio. En el caso de la escala tipo Likert empleada para variables psicológicas se dividieron, en primer lugar, las afirmaciones correspondientes a beneficios en un grupo y las barreras en otro posteriormente se sumaron los puntajes obtenidos en cada grupo; para la categoría de los beneficios un resultado superior a 81 puntos representó un alto nivel de percepción y para las barreras un total superior a 39 estableció un alto nivel de percepción para esta categoría.

Para el análisis univariado y por subgrupos de los datos, las variables cuantitativas fueron resumidas en Mediana con rango intercuartílico para distribuciones heterogéneas de los datos; la variable de interés y otras de naturaleza categórica fueron presentadas como valores absolutos y proporciones con su respectivo intervalo de confianza del $95 \%$ de forma general y distribuidas por sexo. Las pruebas de significancia estadísticas utilizadas fueron Chi cuadrado de Pearson para el contraste de dos variables categóricas y para la relación entre la edad cuantitativa y el nivel de actividad física practicada se aplicó el test de U. de Mann Whitney. La medida de asociación calculada para explorar la relación entre los factores sociodemográficos y los niveles de percepción de beneficios y barreras con la práctica de actividad física global en la población de interés fue el Odds Rattio (OR) crudo con su respectivo intervalo de confianza al 95\% y se empleó la regresión logística como técnica de análisis multivariado para el control de los posibles factores de confusión (OR ajustado) como el sexo y la edad.

La asociación de interés en el análisis multivariado fue la de sexo y nivel de actividad física. Para el ingreso de las demás variables explicativas al modelo de regresión logística se tuvieron en cuenta aquellas con una prueba de significancia de hasta 0,25 . Un cambio del $10 \%$ o más en el OR ajustado al excluir una determinada variable la consideró como factor de confusión y de esta manera se ingresaba nuevamente en el modelo de regresión logística.

La variable "edad" fue recategorizada de acuerdo a los grupos de edad establecidos por el Ministerio de Salud de Colombia (18-26 años/27-59 años/60 años y más) y 
el estrato de la vivienda por niveles socioeconómicos (bajo (1 y 2), medio (3 y 4), alto (5 y 6)).

La variable de nivel de práctica de actividad física fue recategorizada teniendo en cuenta que los físicamente inactivos fueron aquellos con nivel de actividad física leve y los físicamente activos los de niveles moderado y vigoroso. Las variables sociodemográficas con más de dos categorías de respuesta fueron dicotomizadas para efecto del cálculo de medidas de asociación y del análisis multivariado.

\section{Resultados}

La muestra calculada fue de 276 participantes y se seleccionó de un total de elegibles de 662 trabajadores. Las personas encuestadas formaron parte de la mayoría de las dependencias de la Caja. La tasa de respuesta durante el proceso de recolección de la información fue del $100 \%$.

Del total de la muestra de adultos trabajadores de la Caja de compensación residentes en la ciudad de Villavicencio para el primer trimestre del año 2017, por cada tres mujeres había un hombre. La mitad de los participantes incluidos en la muestra tenían 35 años cumplidos o menos (Rango intercuartílico RIC $=28,7-41,8)$. De cada cinco personas incluidas en el estudio cuatro cursaron carreras técnicas y/o universitarias. El nivel socioeconómico medio fue más prevalente en los hombres (66,7\%). El estado civil más prevalente en las mujeres fue ser soltera $(31,9 \%)$. Los hogares unipersonales tuvieron una prevalencia considerablemente más alta en hombres $(12,1 \%)$ que en mujeres (4,8\%) Tabla 1.

Del total de la muestra estudiada, sólo el $33,7 \%$ se consideró activo físicamente (IC ${ }_{95 \%}: 28,1 \%-39,6 \%$ ), proporción que fue significativamente superior en los hombres $(45,5 \%$ frente a $30 \% ; \mathrm{p}=0,009)$.

La mediana del tiempo diario de realización de actividad física total, es decir, la realizada en los tres niveles de intensidad en la muestra de estudio fue de 22,5 minutos (RIC 10-70). Este tiempo fue mayor en los hombres (47,5 minutos/día $(\mathrm{RIC}=20-120)$ frente a 20 minutos/día $(\mathrm{RIC}=0-53,8)$ ). De acuerdo al grupo de edad, la mediana del tiempo diario de realización de actividad física total fue más alta en la muestra de participantes mayores de 60 años (35 minutos/día $(\mathrm{RIC}=5-102,5))$ y en los jóvenes (30 minutos /día $(\mathrm{RIC}=15-120))$.
Tabla 1. Características sociodemográficas de la muestra de trabajadores de la Caja de compensación, Villavicencio, 2017.

\begin{tabular}{|c|c|c|}
\hline VARIABLE & $\mathbf{n}$ & $\begin{array}{l}\text { Porcentaje } \\
\%\end{array}$ \\
\hline \multicolumn{3}{|l|}{ Sexo } \\
\hline Mujer & 210 & 76,1 \\
\hline Hombre & 66 & 23,9 \\
\hline \multicolumn{3}{|l|}{ Grupo de edad ${ }^{a}$} \\
\hline 18-26 años (Juventud) & 49 & 17,8 \\
\hline 27-59 años (Adultez) & 221 & 80,1 \\
\hline 60 años y más (Adulto mayor) & 6 & 2,1 \\
\hline \multicolumn{3}{|l|}{ Nivel de escolaridad } \\
\hline Tecnólogo/Universitario/Postgrado & 233 & 84,4 \\
\hline Secundaria completa & 40 & 14,5 \\
\hline Secundaria incompleta & 2 & 0,7 \\
\hline Primaria incompleta & 1 & 0,4 \\
\hline \multicolumn{3}{|l|}{ Nivel socioeconómico } \\
\hline Bajo (estratos 1 y 2 ) & 99 & 35,9 \\
\hline Medio (estratos 3 y 4) & 175 & 63,4 \\
\hline Alto (estratos 5 y 6 ) & 2 & 0,7 \\
\hline \multicolumn{3}{|l|}{ Estado civil actual } \\
\hline Soltero(a) & 92 & 33,3 \\
\hline Casado(a) & 85 & 30,8 \\
\hline Unión libre & 78 & 28,3 \\
\hline Separado(a)/Divorciado(a) & 15 & 5,4 \\
\hline Viudo(a) & 6 & 2,2 \\
\hline \multicolumn{3}{|l|}{ Composición del hogar } \\
\hline $\begin{array}{l}\text { Integrado por adultos y menores de } 18 \\
\text { años }\end{array}$ & 165 & 59,8 \\
\hline Integrado solo por adultos & 111 & 40,2 \\
\hline \multicolumn{3}{|c|}{ Número de personas que integran el hogar } \\
\hline 1 & 18 & 6,5 \\
\hline De 2 a 4 & 207 & 75 \\
\hline De 5 a 6 & 46 & 16,7 \\
\hline 7 o más & 5 & 1,8 \\
\hline \multicolumn{3}{|l|}{ Tipo de hogar } \\
\hline Nuclear & 198 & 71,7 \\
\hline Extensa & 26 & 9,4 \\
\hline Pareja sin hijos & 22 & 8 \\
\hline Solo una persona & 18 & 6,5 \\
\hline Compuesta & 12 & 4,4 \\
\hline
\end{tabular}

${ }^{a}$ Grupos de edad según ciclo vital establecidos por el Ministerio de Salud de Colombia.

No se encontraron diferencias estadísticamente significativas en la práctica de actividad física según el grupo de edad $(p=0,664)$, el nivel de escolaridad $(p=$ 
0,218), el nivel socioeconómico ( $\mathrm{p}=0,218)$, el estado civil $(\mathrm{p}=0,591)$, la composición del hogar $(\mathrm{p}=0,096)$, el número de personas que integran el hogar $(\mathrm{p}=0,952)$ y el tipo de familia $(p=0,519)$.

La barrera de salud física más prevalente reportada en ambos sexos fue la presencia de dolor osteoarticular (hombres 13,6\%, mujeres 21,9\%). En el grupo de edad de 18 a 26 años la principal barrera fue el dolor precordial al hacer actividad física $(26,7 \%)$ y en los participantes de 27 a 59 años fue la presencia de otro tipo de razones médicas para no realizar actividad física (86,7\%) Tabla 2.

Los beneficios psicológicos percibidos con mayor frecuencia en la muestra de estudio fueron los relacionados con la salud física $(98,5 \%)$ y cardiovascular (98,5\%), seguido de los beneficios relacionados con el mejoramiento de la apariencia física $(98,2 \%)$, el incremento del tono muscular $(98,1 \%)$ y el mejoramiento de la capacidad física $(97,8 \%)$.

Tabla 2. Prevalencia de barreras de salud percibidas para la actividad física en la muestra de trabajadores de la Caja de compensación, Villavicencio, 2017.

\begin{tabular}{|c|c|c|c|}
\hline Variable & n & $\%$ & IC 95\% ${ }^{a}$ \\
\hline Presencia de problemas osteoarticulares & 55 & 19,9 & $15-24,8$ \\
\hline Pérdida del equilibrio debido a mareo & 39 & 14,1 & $9,8-18,4$ \\
\hline Presencia de dolor precordial durante el ejercicio & 30 & 10,9 & $7-14,7$ \\
\hline Otras razones médicas para no hacer ejercicio & 30 & 10,9 & $7-14,7$ \\
\hline Presencia de dolor precordial en reposo & 22 & 8,0 & $4,6-11,3$ \\
\hline Presencia de enfermedad del corazón diagnosticada por profesional médico & 14 & 5,1 & $2,3-7,8$ \\
\hline Consumo actual de medicamentos para la presión sanguínea o afección del corazón & 14 & 5,1 & $2,3-7,8$ \\
\hline
\end{tabular}

a Intervalo de confianza de la proporción al 95\%.

Las principales barreras psicológicas percibidas fueron aquellas relacionadas con la presencia de fatiga $(51,5 \%)$ y el cansancio físico ( $42 \%$ ) seguida de la falta de tiempo $(40,6 \%)$, la distancia de los lugares deportivos $(34,7 \%)$ y los horarios no convenientes de dichos espacios para realizar la actividad física $(34,4 \%)$.

De acuerdo al sexo, los hombres percibieron con mayor frecuencia beneficios relacionados con el mejoramiento de la salud general como el incremento de la fuerza muscular y el funcionamiento general del cuerpo, mientras que en el caso de las mujeres fueron los relacionados con la autopercepción de la imagen corporal. No se encontraron asociaciones significativas en el nivel de percepción de beneficios y barreras para la práctica de actividad física de acuerdo al sexo de los participantes.

La Tabla 3 mostró que la oportunidad de ser inactivo físicamente en las mujeres fue aproximadamente dos veces la oportunidad de ser inactivo en los hombres de la muestra estudiada $\left(\mathrm{OR}=1,940, \mathrm{IC}_{95 \%}: 1,10-3,43\right)$. Dicha asociación se mantuvo al ajustar por las variables de edad y nivel de percepción de beneficios Tabla 4 . Para las demás variables sociodemográficas no se encontraron asociaciones significativas con respecto al nivel de actividad física practicada.

\section{Discusión}

La presente investigación se constituyó como el primer trabajo que estimó la prevalencia de la práctica de actividad física y de los principales beneficios y barreras a nivel físico y psicológico en la población de adultos trabajadores de una Caja de compensación familiar en toda la Orinoquía colombiana.

La prevalencia de práctica de actividad física reportada en este estudio fue significativamente inferior a la reportada por la ENSIN $2010(53,5 \%)^{5}$; En este sentido, Bones-Rocha, et al. ${ }^{25}$ resaltaron que el desgaste y agotamiento físico, mental y social que puede generar para el empleado el desarrollo de su jornada de trabajo influye en gran medida y casi que, de forma inevitable sobre sus estilos de vida, entre ellos, en la reducción en la práctica de actividad física realizada.

Otros estudios nacionales que reportaron prevalencias de práctica de actividad física superiores a la de la presente investigación fueron el realizado a trabajadores de una institución prestadora de servicios de salud de Popayán en el 2007 (43,7\%) y el estudio multinivel para el diagnóstico de la actividad física en población adulta en tres regiones colombianas $(40,7 \%)^{4,26}$. 
Los estudios realizados durante el año 2014 en empleados de una universidad privada de Medellín y en el 2003 a una muestra de adultos de Bogotá mostraron prevalencias de práctica de actividad física cercanas al del presente estudio ( $29 \%$ y $36,8 \%$, respectivamente), empleando el mismo instrumento para su medición
(IPAQ versión corta), siendo mayor el porcentaje en adultos varones ${ }^{2,3}$. Gonzáles, et al. ${ }^{27}$ afirmaron que los participantes con más bajo nivel socioeconómico presentaron menores niveles de actividad física mientras que en el presente estudio los participantes con estratos 1 y 2 fueron quienes reportaron una mayor prevalencia.

Tabla 3. Asociación cruda entre la práctica de actividad física y las variables de interés en la muestra de trabajadores de la Caja de compensación, Villavicencio, 2017.

\begin{tabular}{|c|c|c|c|c|c|c|}
\hline \multicolumn{2}{|l|}{ Variables } & \multirow{2}{*}{$\begin{array}{c}\text { Inactivos } \\
147\end{array}$} & \multirow{2}{*}{$\frac{\text { Activos }}{63}$} & \multirow{3}{*}{$\begin{array}{l}\mathbf{O R}^{\mathbf{a}} \\
1,940\end{array}$} & \multirow{3}{*}{$\frac{\text { IC95\% }^{\mathbf{b}}}{1,10-3,43}$} & \multirow{3}{*}{$\begin{array}{c}\text { Valor p } \\
0,021\end{array}$} \\
\hline \multirow{2}{*}{ Sexo } & Mujeres & & & & & \\
\hline & Hombres & 36 & 30 & & & \\
\hline \multirow{2}{*}{ Edad } & 27 años y más & 154 & 73 & \multirow{2}{*}{1,450} & \multirow{2}{*}{$0,77-2,74$} & \multirow{2}{*}{0,245} \\
\hline & 18-26 años & 29 & 20 & & & \\
\hline \multirow{2}{*}{ Estado Civil } & Sin cónyuge & 77 & 36 & \multirow{2}{*}{1,15} & \multirow{2}{*}{$0,69-1,92$} & \multirow{2}{*}{0,591} \\
\hline & Con cónyuge & 106 & 57 & & & \\
\hline \multirow{2}{*}{ Nivel de escolaridad } & Educación superior & 158 & 75 & \multirow{2}{*}{1,52} & \multirow{2}{*}{$0,78-2,95$} & \multirow{2}{*}{0,218} \\
\hline & Básica y Media & 25 & 18 & & & \\
\hline \multirow{2}{*}{ Nivel Socioeconómico } & Medio y Alto & 122 & 55 & \multirow{2}{*}{1,38} & \multirow{2}{*}{$0,83-2,31$} & \multirow{2}{*}{0,218} \\
\hline & Bajo & 61 & 38 & & & \\
\hline \multirow{2}{*}{ Tipo de Hogar } & Otras familias & 54 & 24 & \multirow{2}{*}{1,2} & \multirow{2}{*}{$0,69-2,11$} & \multirow{2}{*}{0,519} \\
\hline & Nuclear & 129 & 69 & & & \\
\hline \multirow{2}{*}{ Número de personas que integran el hogar } & 5 y más & 34 & 17 & \multirow{2}{*}{1,02} & \multirow{2}{*}{$0,54-1,94$} & \multirow{2}{*}{0,952} \\
\hline & De 1 a 4 & 149 & 76 & & & \\
\hline \multirow{2}{*}{ Composición del hogar } & Solo por adultos & 80 & 31 & \multirow{2}{*}{1,55} & \multirow{2}{*}{$0,92-2,61$} & \multirow{2}{*}{0,096} \\
\hline & Adultos y menores de 18 años & 103 & 62 & & & \\
\hline Nivelde nerconción de Peneficios & Bajo & 17 & 3 & 307 & 0891077 & 0066 \\
\hline viver ae percepcion ae denencios & Alto & 166 & 90 & 3,01 & $0,00-10,11$ & 0,000 \\
\hline Nivel de norcención de Rarraras & Bajo & 169 & 88 & 0.60 & 024107 & 0.181 \\
\hline Niver de percepcion de barreras & Alto & 14 & 5 & 0,09 & $0,24-1,91$ & 0,481 \\
\hline
\end{tabular}

${ }^{a}$ Odds Rattio. ${ }^{\mathrm{b}}$ Intervalo de confianza del OR al 95\%. ${ }^{\mathrm{c}}$ Las variables seleccionadas para ingresar a la regresión logística fueron aquellas con una prueba de significancia de hasta $25 \%$ según criterio de Hosmer Lemeshow.

Tabla 4. Factores asociados a la práctica de actividad física en la muestra de trabajadores de la Caja de compensación, Villavicencio, 2017.

\begin{tabular}{|c|c|c|c|}
\hline Covariables & $\mathrm{OR}^{\mathrm{a}}$ & IC $95 \%{ }^{b}$ & Valor $\mathbf{p}$ \\
\hline $\begin{array}{l}\text { Sexo: } \\
\text { Mujeres } \\
\text { Hombres }\end{array}$ & 1,971 & $1,108-3,505$ & 0,021 \\
\hline $\operatorname{Edad}^{d}$ (años) & 0,978 & $0,953-1,003$ & 0,088 \\
\hline $\begin{array}{l}\text { Nivel de percepción o } \\
\text { beneficios }^{\mathrm{d}} \text { : } \\
\text { Bajo }^{\mathrm{c}} \\
\text { Alto }\end{array}$ & 3,520 & $0,980-12,645$ & 0,054 \\
\hline
\end{tabular}

Con relación a las principales barreras de salud para la práctica de actividad física, la proporción de participantes en el presente estudio que manifestaron 42 alguna enfermedad del sistema osteoarticular (19,9\%) fue similar a la reportada por el estudio de Martínez, et al. ${ }^{7}(18 \%)$ e inferior a la reportada por Rodríguez, et al $(25 \%)^{28}$. El estudio titulado "Salud pública y actividad física de los mayores de Extremadura" mostró una prevalencia significativamente mayor de presencia de enfermedades del corazón $(19,4 \%)$ en comparación con la obtenida en el presente trabajo $(5,1 \%)^{29}$.

En la muestra estudiada se reportaron altos niveles de percepción de beneficios psicológicos para la práctica de actividad física en ambos sexos (hombres 92,4\%, mujeres 92,9\%), proporciones similares a las encontradas por Becerra, et al. ${ }^{30}$ en su investigación. En el presente trabajo se encontró un mayor porcentaje de percepción de beneficios en las personas sedentarias que en participantes físicamente activos, lo cual corroboró el hecho de que aunque las dos terceras partes de los encuestados no realizan actividad física 
de forma constante, la mayoría de ellos sí reconocían las bondades que puede traer el ejercicio para su salud.

Los beneficios psicológicos percibidos más prevalentes en el presente trabajo estuvieron relacionados con el mejoramiento del estado de salud física y cardiovascular y la autopercepción de la imagen corporal. En otros estudios como el desarrollado en población adulta de un municipio antioqueño se reportaron proporciones mayores de percepción de beneficios con relación al disfrute de dicha práctica y, en segundo lugar, los relacionados con la promoción de un estado de salud general más deseable ${ }^{31}$. Rodríguez, et al en su estudio realizado en adultos madrilenses encontraron como principales beneficios percibidos para la práctica de actividad física la diversión (29,9\%), el mantenimiento de la forma $(26,4 \%)$ y el mejoramiento del estado de la salud $(16,1 \%)^{32}$.

Las principales barreras psicológicas percibidas fueron las relacionadas con las sensaciones de fatiga $(51,5 \%)$ y cansancio $(42 \%)$ que puede generar la práctica de actividad física, seguida de la falta de tiempo (40,6\%) y la ausencia de espacios cercanos para hacer ejercicio $(34,7 \%)$. En este sentido, Castro, et $\mathrm{al}^{31}$ reportaron como principales barreras la falta de tiempo (64\%) y la carencia de infraestructuras apropiadas para la práctica deportiva (62\%). La ausencia de tiempo y de lugares apropiados para realizar ejercicio también fueron las barreras más importantes reportadas por Seclén, et al. ${ }^{33}$. Mantilla también reportó como principales barreras para la práctica de actividad física la falta de tiempo y de voluntad en su estudio realizado en adultos de una localidad bogotana ${ }^{34}$.

En esta investigación se encontró que las mujeres de la muestra tuvieron una mayor oportunidad de ser inactivas físicamente en comparación con sus compañeros del sexo opuesto cuando se ajustó por edad y nivel de percepción de beneficios. Gómez, et $\mathrm{al}^{3}$ reportaron similares resultados al demostrar que los hombres presentaban una mayor oportunidad de realizar actividad física (OR = 1,62 IC 95\% 1,31-2,01) cuando se ajustó por varios factores de confusión, entre ellos la edad, el estado civil y el nivel educativo. De la misma manera, Zafra, et $\mathrm{al}^{35}$ encontraron mayores niveles de sedentarismo en mujeres $(\mathrm{OR}=2,13$ IC $95 \%$ 2,03-2,23). Meseguer, et $\mathrm{al}^{36}$ reportaron una mayor oportunidad de realizar actividad física en los hombres al ajustarse por las variables de edad, nivel de escolaridad e índice de masa corporal $(\mathrm{OR}=2,41 \mathrm{IC}$ 95\%: 2,20-2,64).
Para la presente investigación, los cuestionarios seleccionados para la estimación del nivel de actividad física practicado y la prevalencia de beneficios y barreras psicológicas y de salud física han sido empleados frecuentemente en este tipo de proyectos, demostrándose en varios de ellos su aceptable grado de fiabilidad y reproducibilidad de los datos recolectados.

Con respecto a la versión corta del IPAQ se reportaron varias dificultades: el nivel de actividad física practicado se obtuvo a partir del reporte entregado durante los últimos siete días, desconociendo otros patrones de ejercicio en periodos de tiempo anteriores; en segundo lugar la versión corta del IPAQ, arroja un nivel de práctica de actividad física de manera global, sin discriminarla por dominio (tiempo libre, recreativo, estudio/trabajo, transporte) por lo que los hallazgos de la presente investigación se limitaron a ser comparados con trabajos que emplearon el mismo instrumento $\mathrm{u}$ otro de características similares; por último y teniendo en cuenta que el cuestionario se diligenció a partir del reporte entregado por el participante, varias de las personas incluidas en la muestra no tuvieron muy claro el proceso de asignación del tiempo destinado a cada uno de los niveles de intensidad de práctica de actividad física, lo que afectaría la confiabilidad de los resultados.

Aunque en el presente trabajo se indagaron sobre aspectos psicológicos y de salud física que podrían tener una asociación con la práctica de actividad física en la población de estudio, las condiciones del tipo de diseño y el hecho de haber recolectado la información en un solo momento dificultaron la estimación de dichas relaciones por cuestiones de temporalidad.

En la muestra de trabajadores estudiada se encontró una baja prevalencia de actividad física, siendo menor en el sexo femenino. Las principales barreras de salud física percibidas para la práctica de actividad física fueron la presencia de problemas osteoarticulares, la pérdida del equilibrio debido a mareo y la presencia de dolor precordial durante la actividad física. Asimismo, las mujeres presentaron un porcentaje mayor de barreras de salud física percibidas que su sexo opuesto. Dentro de los principales beneficios identificados para la práctica de actividad física se encontraron los relacionados con el mejoramiento del estado de salud y la autopercepción de la imagen corporal, mientras que las principales barreras reportadas fueron las relacionadas con la sensación de agotamiento, la falta de tiempo y la ausencia de espacios apropiados para la práctica de ejercicio. Por último, se encontró que la oportunidad de 
ser inactiva físicamente en las mujeres fue dos veces la oportunidad de los hombres al ajustarse por las variables edad y nivel de percepción de beneficios.

Los hallazgos encontrados en la actual investigación plantearon puntos interesantes para la planeación de otro tipo de estudios como lo fue el hecho de que las personas inactivas físicamente sean aquellas que reportaron niveles más altos de percepción de beneficios psicológicos. De igual forma sería de utilidad establecer, la asociación existente entre el nivel de ejercicio realizado por los empleados y las variables relacionadas con la productividad laboral, tasas de incapacidades y las proporciones de accidentes de trabajo y enfermedades laborales.

\section{Agradecimientos}

Se agradece la valiosa colaboración por parte del director administrativo, el jefe de la división de gestión humana y de la gestora de salud y seguridad en el trabajo de la Caja de compensación familiar de la ciudad de Villavicencio y por parte del director de proyecto, cuyo apoyo fue fundamental para la ejecución del presente estudio.

\section{Consideraciones éticas}

Según la Resolución 8430 del año 1993 del Ministerio de Salud el estudio planeado se consideró de riesgo mínimo. El protocolo de investigación contó con la aprobación por parte del comité de ética de la Universidad de los Llanos. Durante la socialización verbal y escrita se le informó a cada participante sobre el grado de confidencialidad de la información manejada, la libre autonomía sobre su participación en el estudio y las no implicaciones que se generarían tras su no participación o desistimiento.

\section{Conflicto de interés}

El investigador principal declara conflicto de intereses ya que para el momento de la realización del presente estudio se encontraba prestando sus servicios profesionales como contratista en la Caja de compensación familiar.

\section{Referencias}

1. Granados C, Rivera D, Muñoz C, Donado C. Documento técnico con los contenidos para el mejoramiento de la gestión territorial de los referentes departamentales, en la promoción de hábitos de vida saludable, con énfasis en alimentación y prevención del consumo de tabaco a través de la práctica regular de la actividad física. 1 ed. Bogotá: Imprenta Nacional; 2011.

2. Suárez GR, Zapata VS, Cardona AJ. Estrés laboral y actividad física en empleados. Divers Perspect Psicol. 2014; 10(1): 131-141.

3. Gómez LF, Duperly J, Lucumí DI, Gámez R, Venegas AS. Nivel de actividad física global en la población adulta de Bogotá (Colombia). Prevalencia y factores asociados. Gac Sanit. 2005; 19(3): 206213. DOI: $10.1157 / 13075953$.

4. Prieto RA, Agudelo CA. Enfoque multinivel para el diagnóstico de la actividad física en tres regiones de Colombia. Rev Salud Pública. 2006; 8(Supp12): 57-68.

5. Ministerio de la Protección Social, Instituto Colombiano de Bienestar Familiar, Instituto Nacional de Salud. Encuesta Nacional de la Situación Nutricional en Colombia ENSIN 2010. 1 ed. Bogotá: Da Vinci \& Cía S en C; 2011.

6. Zamora MM, Cruz AC. El papel del tiempo libre en la salud de un grupo de conductores de carga. Salud Trabaj. 2011; 19(1): 47-56.

7. Martínez LE, Saldarriaga JF. Inactividad física y ausentismo en el ámbito laboral. Rev Salud Pública. 2008; 10(2): 227-238. DOI: 10.1590/S012400642008000200003.

8. Hamer M, Stamatakis E, Steptoe A. Dose-response relationship between physical activity and mental health: the Scottish Health Survey. Br J Sports Med. 2008; 43(14): 1111-1114. DOI: 10.1136/ bjsm.2008.046243.

9. Ramos PM. Enfoque para justificar la promoción y prevención como medios en la erradicación del sedentarismo en el ámbito laboral. Salud Trabaj. 2007; 15(2): 119-128.

10. Hoffmeister L, Vidal C, Vallebuona C, Ferrer N, Vásquez $P$, Núñez G. Factores asociados a accidentes, enfermedades y ausentismo laboral: análisis de una cohorte de trabajadores formales en Chile. Cienc Trab. 2014; 16(49): 21-27. DOI: 10.4067/S0718-24492014000100005.

11. Decreto por el cual se determina la organización y administración del Sistema General de Riesgos Profesionales. Decreto 1295/1994 de 22 de junio. Diario oficial $n^{\circ} 41.405$ (24 Jun 1994).

12. Ley por la cual se dictan disposiciones para el fomento del deporte, la recreación, el aprovechamiento del tiempo libre y la Educación Física y se crea el Sistema Nacional del Deporte. Ley 181/1995 de 18 de enero. Diario oficial n 41.679 (18 Ene 1995).

13. Ley por la cual se introducen reformas al Código Sustantivo del Trabajo y se dictan otras 
disposiciones. Ley 50/1990 de 28 de diciembre. Diario oficial n 39.618 (1 Ene 1991).

14. Ley por la cual se modifica el régimen del Subsidio Familiar y se dictan otras disposiciones. Ley 21/1982 de 22 de enero. Diario oficial n 35.939 (5 Feb 1982).

15. Ortiz AL. Estadísticas poblacionales Cofrem enero 2017 [CD-ROM]. Villavicencio; 2017.

16. Patterson E. Guidelines for Data Processing and Analysis of the International Physical Activity Questionnaire (IPAQ) - Short and Long Forms. 1 ed. Ginebra: IPAQ Core group; 2005.

17. Thomas S, Reading J, Shephard RJ. Revision of the Physical Activity Readiness Questionnaire (PAR-Q). Can J Sport Sci. 1992; 17(4): 338-345.

18. Fonseca J, Barbosa L, Almeida PC. Reliability and validity of the Exercise Benefits/Barriers scale in the elderly. Acta Paul Enferm. 2012; 25(1): 48-53. DOI: $10.1590 / \mathrm{S} 0103-21002012000800008$.

19. Mantilla SC, Gómez A. El cuestionario internacional de actividad física. Un instrumento adecuado en el seguimiento de la actividad física poblacional. Rev Iberoam Fisioter Kinesol. 2007; 10(1): 48-52. DOI: 10.1016/S1138-6045(07)73665-1.

20. Brown WJ, Trost SG, Bauman A, Mummery K, Owen N. Test-retest reliability of four physical activity measures used in population surveys. J Sci Med Sport. 2004; 7(2): 205-215.

21. Craig CL, Marshall AL, Sjostrom M, Bauman AE, Booth ML, Ainsworth BE, et al. International physical activity questionnaire: 12-Country reliability and validity. Med Sci Sports Exerc. 2003; 35(8): 1381-1395. DOI: 10.1249/01. MSS.0000078924.61453.FB.

22. Rodríguez FA. Cuestionario de aptitud para la actividad física (C-AAF), versión castellana del PAR-Q revisado. Apunts. 1994; 31: 301-310.

23. Sechrist KR, Walker SN, Pender NJ. Development and psychometric evaluation of the exercise benefits/ barriers scale. Res Nurs Health. 1987; 10(6): 357365. DOI: 10.1002/nur.4770100603.

24. Nies A, Jones M. The relationship of perceived benefits of and barriers to reported exercise in older African American woman. Public Health Nurs. 1994; 13(2): 151-158. DOI: 10.1111/j.15251446.1996.tb00233.

25. Bones K, Muntaner C, González M, Bernales P, Vallebuona C, Borrell C, et al. Clase social, desigualdades en salud y conductas relacionadas con la salud de la población trabajadora en Chile. Rev Panam Salud Publica. 2013; 33(5): 340-348.

26. Díaz J, Muñoz J, Sierra C. Factores de riesgo para enfermedad cardiovascular en trabajadores de una institución prestadora de servicios de salud, Colombia. Rev Salud Públic. 2007; 9(1): 64-75.

27. González S, Sarmiento O, Lozano O, Ramírez A, Grijalba C. Niveles de actividad física de la población colombiana: desigualdades por sexo y condición socioeconómica. Biomédica. 2014; 34(1): 447-459. DOI: https://doi.org/10.7705/biomedica. v34i3.2258

28. Rodríguez MC, Molina J, Jiménez C, Pinzón T. Calidad de vida y actividad física en estudiantes, docentes y administrativos de una universidad de Bogotá. Cuadernos Hispanoam Psicología. 2011; 11(1): 19-37.

29. Gusi N, Madruga M, Prieto J. Salud pública y actividad física de los mayores en Extremadura. 1 ed. Extremadura: Junta de Extremadura; 2009.

30. Becerra M, Díaz L. Niveles de actividad física, beneficios, barreras y autoeficacia en un grupo de empleados oficiales. Av Enferm. 2008; 26(2): 43-50.

31. Castro J, Patiño F, Cardona B, Ochoa V. Aspectos asociados a la actividad física en el tiempo libre en la población adulta de un municipio antioqueño. Rev Salud Pública. 2008; 10(5): 679-690.

32. Rodríguez G, Boned C, Garrido M. Motivos y barreras para hacer ejercicio y practicar deportes en Madrid. Rev Panam Salud Pública. 2009; 26(3): 244-254.

33. Seclén JA, Jacoby ER. Factores sociodemográficos $\mathrm{y}$ ambientales asociados con la actividad física deportiva en la población urbana del Perú. Rev Panam Salud Pública. 2003; 14(4): 255-264.

34. Mantilla S. Actividad física en habitantes de 15 a 49 años de una localidad de Bogotá, Colombia, 2004. Rev Salud Pública. 2006; 8(2): 69-80.

35.Zafra JH, Millones E, Retuerto MA. Factores sociodemográficos asociados a actividad física y sedentarismo en población peruana adulta. Rev Peru Epidemiol. 2013; 17(3): 1-6.

36. Meseguer C, Galán I, Herruzo R, Zorrila B, Rodríguez F. Actividad física de tiempo libre en un país mediterráneo del sur de Europa: adherencia a la recomendaciones y factores asociados. Rev Esp Cardiol. 2009; 62(10): 1125-1133. DOI: 10.1016/ S0300-8932(09)72381-4. 